\title{
Design and Implement a Smart Computer Lab for Exam and e-Learning Issue
}

\author{
Suhail N. Shahab ${ }^{1}$, Raid W. Daoud ${ }^{2}$, Ihab A. Satam ${ }^{3}$ \\ 68suhe1@gmail.com ${ }^{1}, \underline{\text { raid.daoud@gmail.com }}{ }^{2}, \underline{\text { ihabengmechatronics@gmail.com }}{ }^{3}$
}

Northern Technical University, Al-Hawija Tech. Institute, Kirkuk, Iraq.

\begin{abstract}
Computer network is important for transferring data through different media to perform a high accuracy function. In this paper a computer network was designed, the network consists of several clients to communicate with each other subjectively to deliver the desired output to a certain point. The ethernet method was used and the base protocol is (IEEE803.2) which depends on the token ring principle in transferring data. The new addition to the network is to connect the network to the internet and control it remotely, where we can perform a test to the students through a site that is designed specifically for the tests. Theoretically, the test was done using Packet trainer software as well as an actual test was done for the students. The report was printed after the student finishes the exam. This network is the typical solution to transform into E-learning at universities and colleges.
\end{abstract}

\section{Introduction}

The incorporation of computer networks and the internet with mobile phone technologies have an impression on the increasing encouragement of information technology in the education system [1]. This is confirmed by the increased enactment of electronic learning (e-learning) worldwide by 2016, which reached nearly $8 \%$ per year [2]. This increase is due to the modernisation of plentiful Learning Management System (LMS) applications, which cultivating benefits of electronic learning for high flexibility learners. Nowadays, LMS offers a more planned and more stretchy learning process. With the swift enlargement of e-learning, security matters still a highly profound issue in its enactment. E-learning system is an online operation that should be secured to avoid swindle and to protect the discretion of users [3][11]. Maintaining the security issues in the enactment of e-learning is very important to form the confidence of its users. Moreover, the eLearning system is an open, distributed and consistent system, therefore, security becomes an important contest to ensure that only the factual people are able to use and entree the system [4].

Therefore, in this paper, a proposed network was developed and implemented using hybrid network topology and devices. The e-exam is one of the most important things in the e-learning field, so, it is achieved using the computer network and smart management of the data transfer. Security is a vigorous issue to be considered for the system which is carried in aggressive condition. Organization of a system in a human non-interposable place is extremely defenseless without security [5]. The Internet of things is ready to connect anyone at any time to anything anytime, 
anywhere. The basic idea is to allow self-determining and confidential communication to exchange of data between real-world devices and applications of the Internet of things [6] [12]. Computer network area requires professionals with solid networking theory and practical hands-on experience. Together with the fast technological advancement in the field of computer networks and Information Technology industry, the need for a large number of skilled network experts also increased [7][8]. Cisco Packet Tracer is an effective education simulation software that supports computer networking students to experiment and practice network tasks. Cisco Networking Academy Program (CNAP) introduced Packet Tracer as a tool for teaching and learning of computer network courses by providing "simulation, visualization, authoring, collaboration capabilities and assessment" [9][10].

\section{Methodology}

The following parts are detailed the proposed network and how to use different configurable places of different purposes.

\subsection{Network Building.}

The wired computer network principle was used since it has high security and high speed. Several types of connections maybe used such as: star, ring and tree connections. In this paper, a Star connection was used since it is easy to gather the computers and install the hubs. Firstly the dimension of the lab as measured and concluded that the lab can have more than 40 computers (client). Figure 1 shows the topology which is used in this work, for which multi types of network devices were used such as a server, client and hub switch.

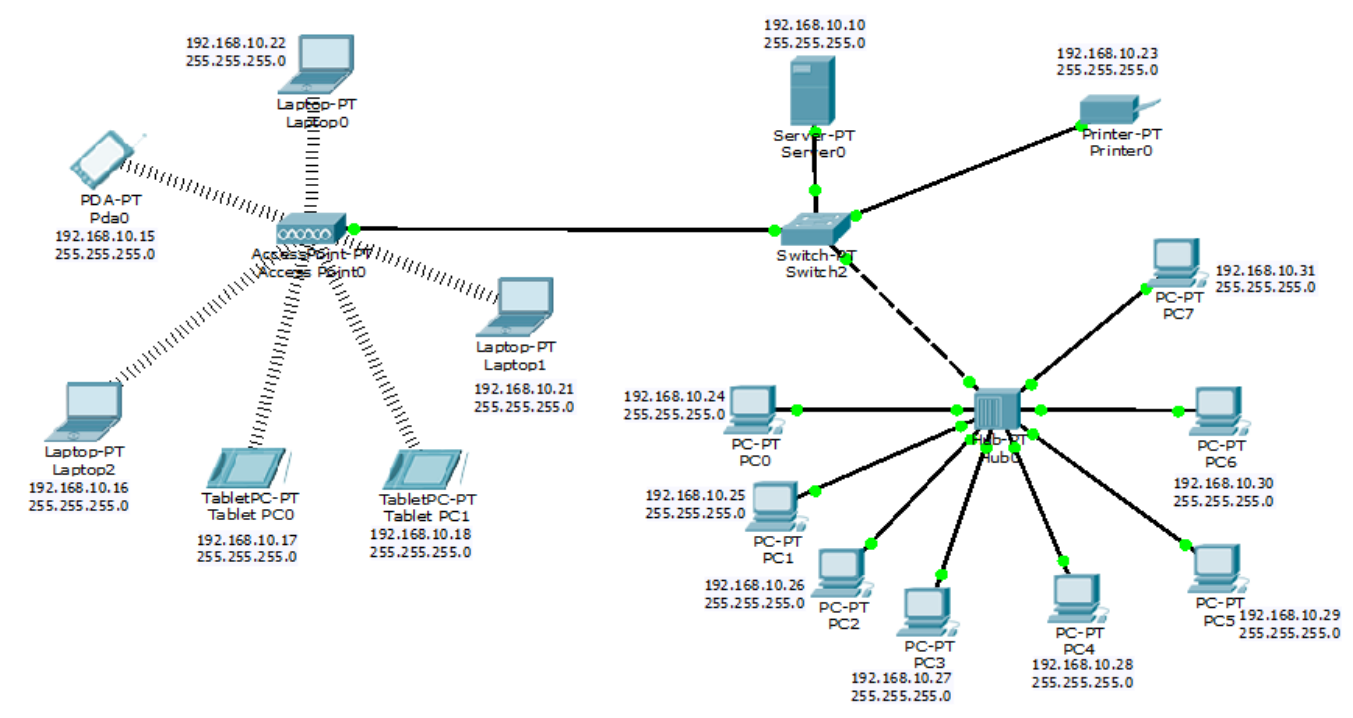


Fig. 1. The proposed network and its topology.

\subsection{Network Parts:}

\section{1- Clients:}

The main part in the network is the computer. Since the aim of this paper is the e-learning, therefore the computer must be used. The universities used different kinds of software such as: Office, Engineering MATLAB, AutoCAD....etc.ad they need computers with special properties for experiments and exams. Nowadays, at every university. Some exams are conducted on computers and that leads to the inevitability of having a computer lab. Thus the network helps with preserving and printing the answers directly after finishing the exam.

\section{2- Wires:}

In this Network, the wires used were the S.T.P ( Shielded Twisted Pair) type, since it can endure the changes in weather like heat, cold and anti-rodent. The longest distance between two points is $180 \mathrm{~m}$ while in the lab the largest distance doesn 't exceed 1 meter also the distance between every computer and the hub is nearly 25 meters. Every end in the wire has Rj45 socket in order to connect the wires with network devices.

\section{3- Connect devices.}

In every network, there are several connecting devices for exchanging data between the network elements. For example, there is a smart distributor device, in which this device makes an address for every element in the network. Other devices are responsible for connecting the network elements electrically such as the Hub Switch. In this paper, an intelligent hub switch is used because of its ability to run the network and preventing the loss of the address in Data transferring. Hub Switch is the joining point where every network elements meet. It joins the Clients, printers and other connecting devices

\section{4- IP address}

One of the important things that must be considered in any computer network design is the IP address. It has many types. In this paper Type $\mathrm{C}$ is used because it is the most common. The router in a computer network has the ability to make an IP for every device connected to the network. But to increase the control over the network, manual IP distribution to ensure that there is no extra unwanted element inside the network that could spy or sabotage the network.

A simulation for the network is made by packet tracer software. IP address was made for every element in the network, a certain data was sent in batches and the process was partially successful, a minor problem appeared in the network when the number of computers exceeds 20 , the distributer start to give a computer address for another purpose thus the result will be wrong for the examination 
process. The problem was solved using another distributer when the number of computers exceeds the limit when IP begins to be lost.

IP address consists of four parts. The first two parts refer to the network address, while the other two refer to the device. All the network elements have the same number for the first two parts when the difference begins to appear in the second two parts. Figure 2 below shows how a message transfer from a computer with ip address 192.168.10.13 to a computer with ip address 192.168.10.21. The figure shows the shortest path for transferring, variables that determine the loss in data, the Time and number of points that the path went through.



Fig. 2. The path of the message across the network.

This computer network was designed with a number of switches as well as printers and scanners. One of the problems faces in this work is that always an exclamation mark appears in the network icon in the computers that connected in the network, see Figure 3 below, this lead to a conclusion that there is a confliction in the network addresses or there is no main server for the network which leads to data been lost. The problem was solved using Home Group for windows system that makes a closed work group that allows only members who have a serial number to access the network.

A new technique added to the network in order to increase the number of computers in another place but connected to the same network. This technology is Internet of Things or IoT. With this technology, more than one workplace is connected to the network. A work group was setup consists of numbers of computers under one server, where the data transferring can be controlled, and by packet tracer, a communication between two ends on the network was done and the process was successfully made. The received data percentage is $100 \%$ and the data lost was almost $0 \%$, the time less than 1ms, see Figure 4.

In computer network there re the wire and wireless type. a merge between the two types obtained using ethernet/WiFi technology. One of the results accomplished in this merge is to use all 
the potential of the network in other fields with the same sort of data. this type of network facilitates the network management and the data receiving from students or employees.

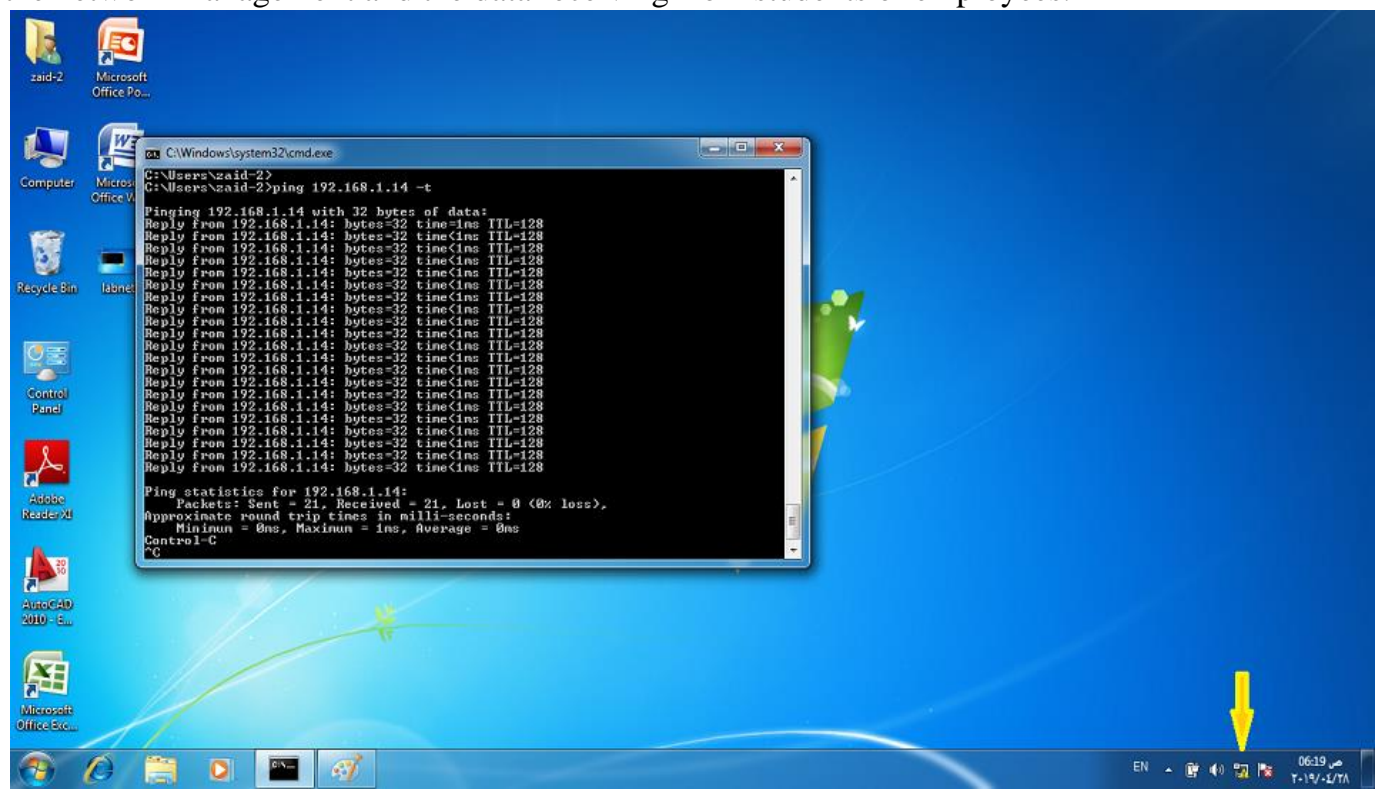

Fig. 3. Exclamation mark problem and ttl for the network.

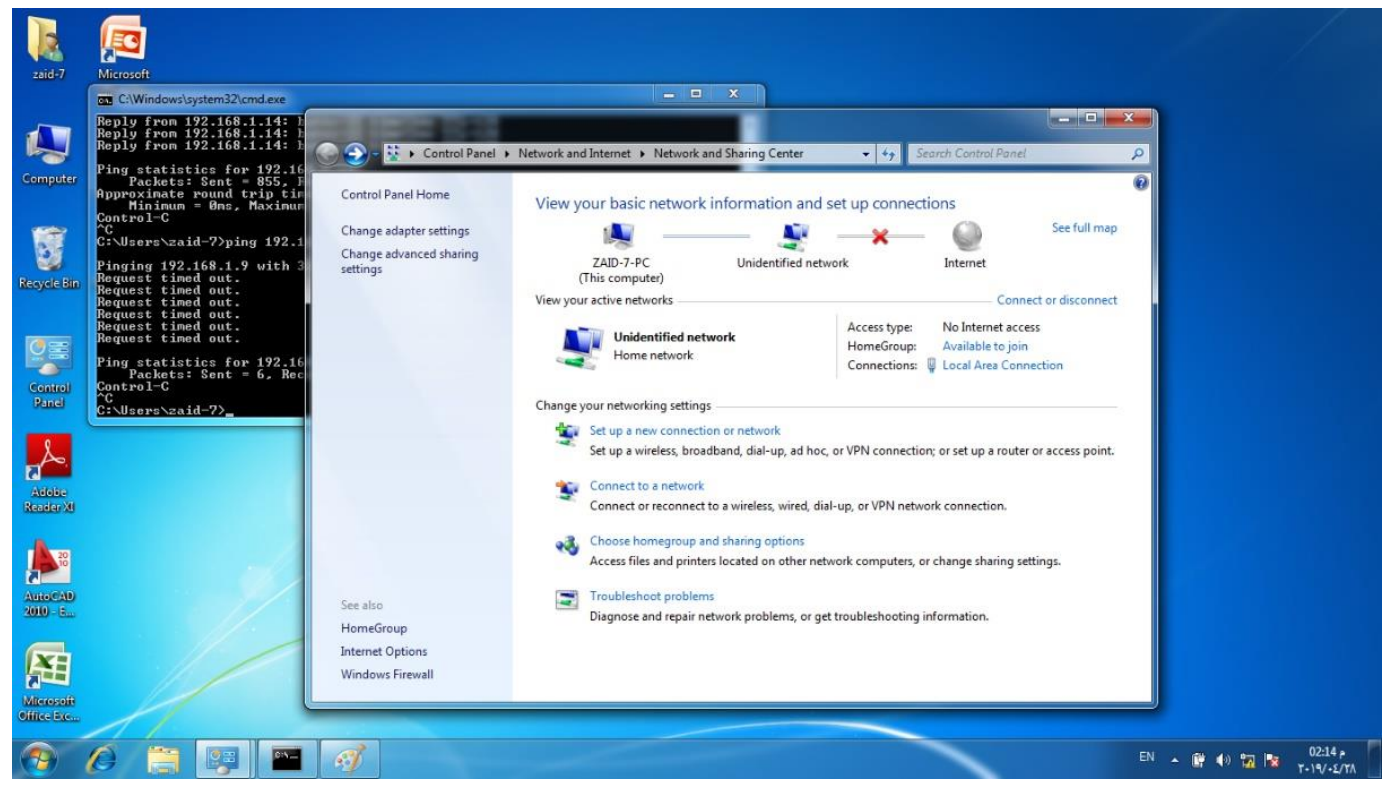


Fig. 4. HomeGroup solution for the best quality of data sharing network.

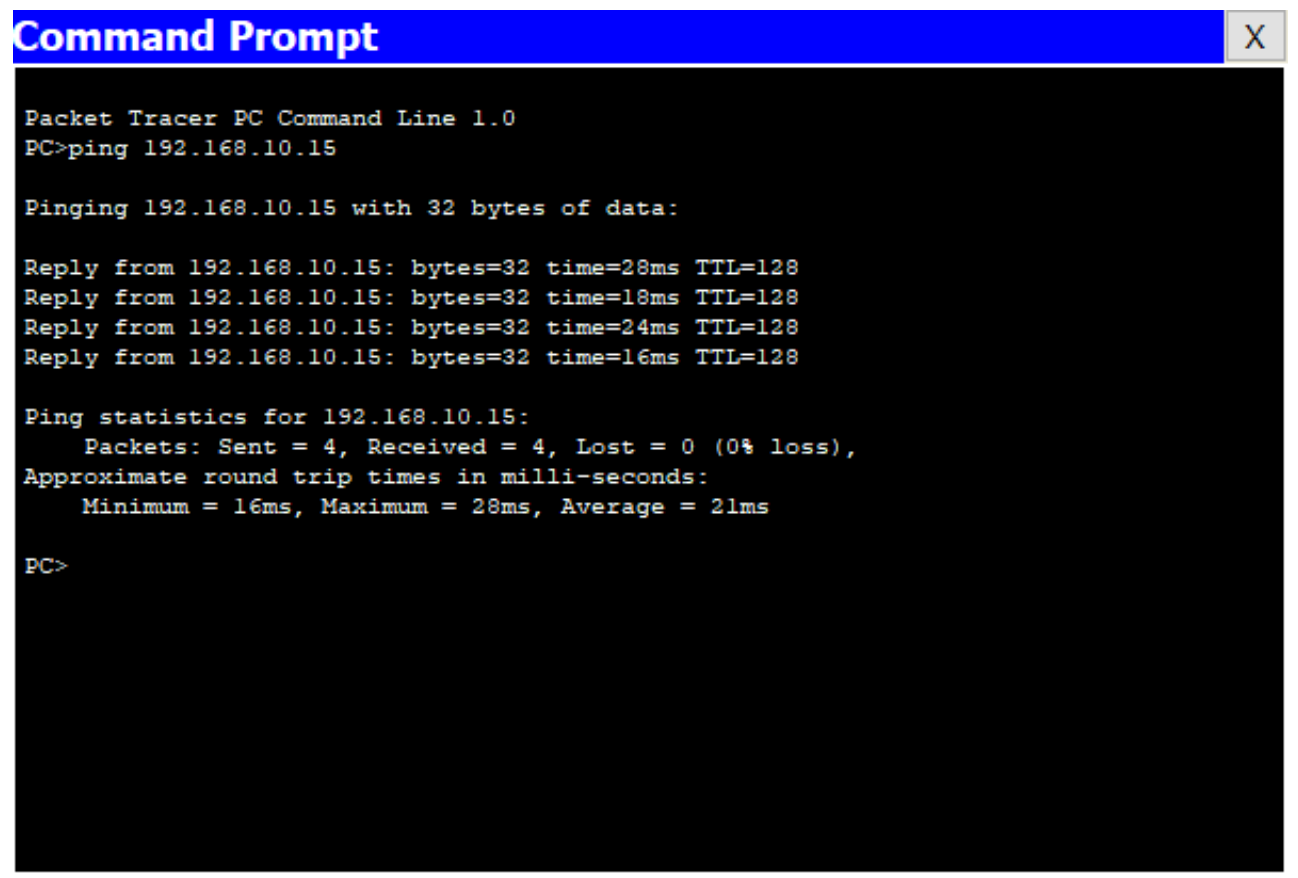

Fig. 5. Simulation $\mathrm{ttl}$ and number of packets send/ receive.

For example there are three computer labs and the task is to perform to execute a test for a group of students distributed in these labs. By using IoT technique, it become very easy to send the students answers to one printer installed in one of these labs. For this the router must be very strong and has the ability to give an IP in every computer connected to the network in spite of there is a fixed IP in the wireless network, but in for the far computers, they will be connected using WIFI to be included in the management process. Another problem faced the work is the hacking for the wireless network since the connection only depends on the serial number of the router, this problem can be solved but in the future work of this research.

\section{Results}

The results were recorded and sketched by means of packet tracer software and from the real implementation of the network. Figure $\mathbf{6}$ shows the connection mechanism of the remote computer with the network, that's how the computers have the ability to transmit, print and execute other function the client desire. 


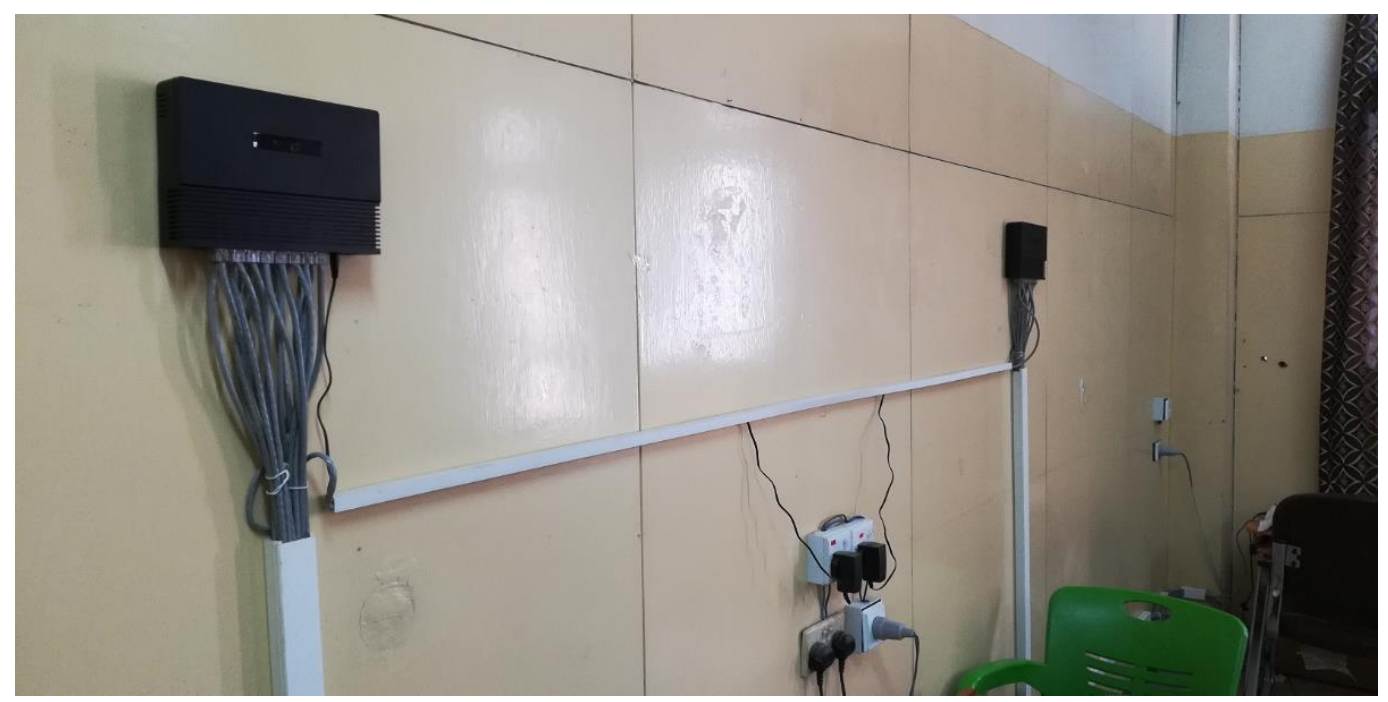

Fig. 6. Physical connection of the network.

Figures 7-8 shows the real network model of the proposed idea with printing test and sharing data. As shown in the mentioned figures, there are no losses in the send data.

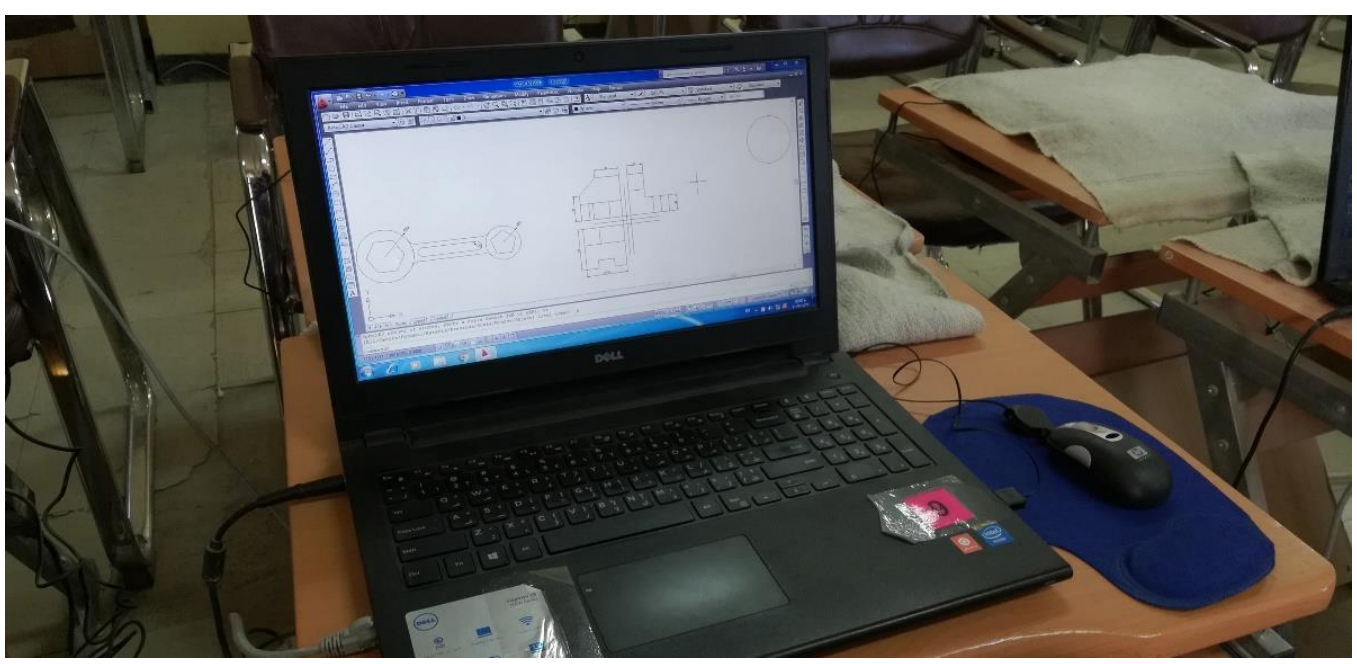

Fig. 7. Preparing to send data to the network printer.

The data transfer are managed automatically by the network protocols, each packet is transferred by any PC client will be addressed to destination address. In this work, an AutoCAD 
software is used to share its data upon the network. Although there were small errors in the network, the reception of the data packet was sent through it.

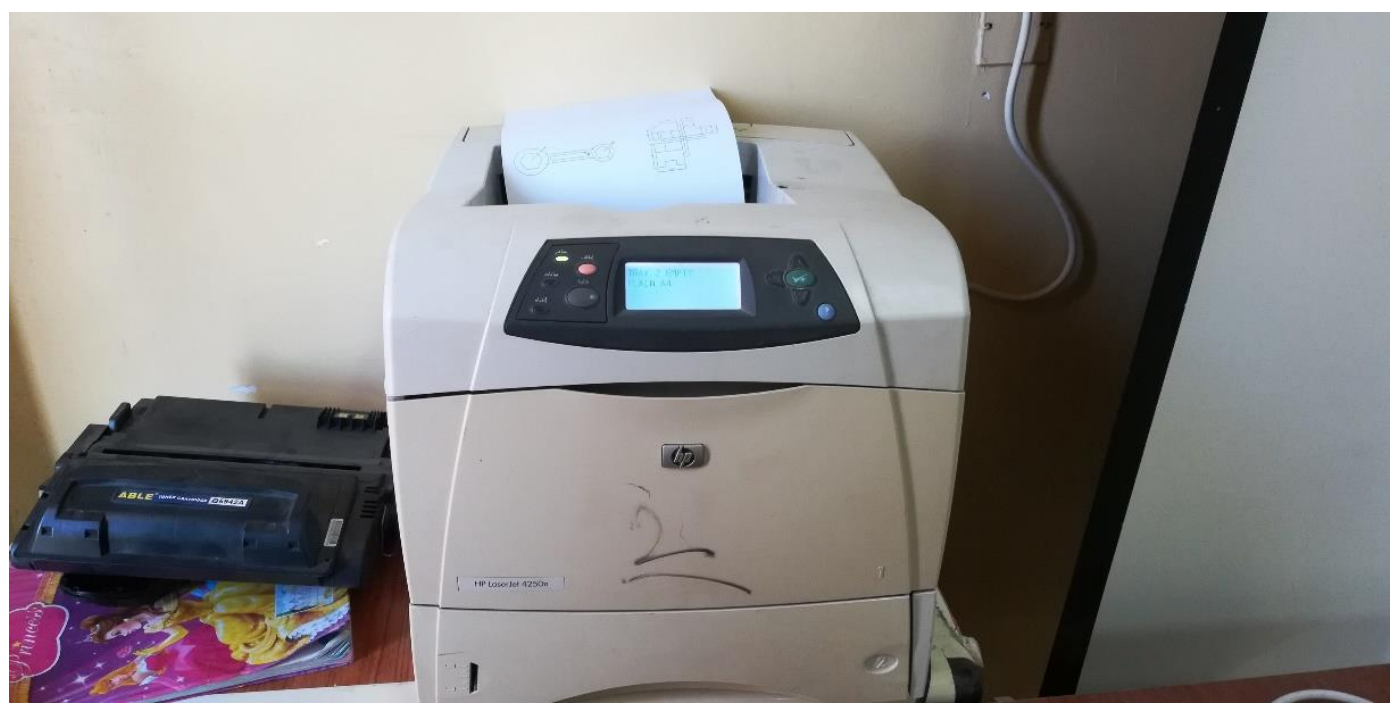

Fig. 8. Data were received bt the network printer, via ethernet or $\mathrm{WiFi}$ is the same.

\section{Conclusion:}

A new mechanism has been found to manage the transfer of student answers if there was a test performed or an administrative process for a company.

It can be concluded that:

1- It is easy to work with data and receive reports.

2- The network can be expanded.

3- In this work the remote user problem was solved to make use of the network resources.

4- Using IOT, it was easy to observe and followed data any where in a certain area through network and Internet

\section{References}

[1] Gede S., Rukmi S. Hartati and et al: Proposed Model For E-Exam Availability In WLAN Environment. ISBN :978-1-5090-2689-0, ICSGTEIS, pp. 89-92 (2016).

[2] A report by Docebo, E-Learning Market Trends \& Forecast 2014 - 2016, Report, March 2014, https://www.docebo.com/landing/contactform/elearning-market-trendsand-forecast-2014-2016-doceboreport.pdf, (diakses 9 Oktober 2015). 
[3] Byeong Ho Kang and Hyejin Kim. Proposal: A Design of E-learning User Authentication Sistem. Vol. 9, No. 1, pp. 45-50. International Journal of Security and Its Applications (2015)

[4] I. Bandara, F. Ioras, K. Maher. Cyber Security Concerns In E-Learning Education. ISBN: 978-84-6172484-0, pp.0728 - 0734. Proceedings of ICERI2014 Conference, Seville, Spain, , 17th-19th November (2014).

[5] Waleed Kh. Alzubaidi and Shaimaa H. Shaker. Methods of Secure Routing Protocol in Wireless Sensor Networks. ISSN (Print): 2074 - 0204, ISSN (Online): 2521 - 3504, Vol. 10 No. 3. Journal of ALQadisiyah for computer science and mathematics (2018)

[6] N.F. Alwan, M.K. AL-Nuaimi. The Role and Important of Internet of Things in Building Sustainable City. Vol. 36, No. 1, pp. 22-29, Engineering and Technology Journal (2018).

[7] J. Crichigno and I. L. Hurtado, "An alternative model for computer networks education in computing disciplines," presented at the American Society for Engineering Education Conference, San Antonio, TX, June 11, (2012).

[8] M. Abdullah and A. Ehsan. Teaching methodologies for computer networks lab. Vol. 5, No. 2, pp. 109119, Int. J. Adv. Sci. and Technical Res. (2012).

[9] Noor Maizura Mohamad Noor, Nadiah Yayao, and Sumazly Sulaiman. Effectiveness of Using Cisco Packet Tracer as a Learning Tool: A Case Study of Routing Protocol. Vol. 8, No. 1. International Journal of Information and Education Technology. January (2018).

[10] Garima Jain, Nasreen Noorani, Nisha Kiran, Sourabh Sharma, "Designing \& simulation of topology network using Packet Tracer", International Research Journal of Engineering and Technology (IRJET), Vol. 2, No. 2, 2015.

[11] Nathaniel S. Tarkaa, Paul I. Iannah and Isaac T. Iber. Design and Simulation of Local Area Network Using Cisco Packet Tracer. Vol. 6, Issue 10, pp. 63- 7, The International Journal of Engineering and Science (IJES), (2017).

[12] Anwar Hossain \& Mahabuba Zannat. Simulation and Design of University Area Network Scenario (UANS) using Cisco Packet Tracer. Vol. 19, Issue 3, Global Journal of Computer Science and Technology: G Interdisciplinary (2019) 